\title{
Reabilitação estética após fratura mandibular unilateral e perda precoce de dentes anteriores: relato de caso
}

\section{Aesthetic rehabilitation after unilateral mandibular fracture and early loss of anterior teeth: a case report}

\author{
Tamara Ripplinger* \\ Gabriela dos Santos Pinto** \\ Deisi Spessato ${ }^{* * *}$ \\ Larissa Corrêa Brusco Pavinato ${ }^{* * * *}$ \\ Caroline Dias Tams Riffel ${ }^{* * * *}$
}

\section{Resumo}

Objetivo: discutir reabilitação de um caso clínico de avulsão precoce de dentes decíduos com fratura mandibular unilateral ocorrida após uma queda de três metros de altura. Relato de caso: paciente do gênero masculino, leucoderma, 2 anos e 5 meses de idade, apresentou-se na emergência do Hospital São Vicente de Paulo na cidade de Passo Fundo - RS com história de queda da laje da casa que estava em reforma. Ao exame físico intraoral, observou-se ausência dos elementos dentários 51 e 61, equimose em região mandibular unilateral e fratura mandibular. Como tratamento adotado utilizou-se um mantedor de espaço estético-funcional após a redução da fratura mandibular. Considerações finais: a ausência dos padrões de sorriso das crianças influencia a saúde e qualidade oral de vida de duas maneiras, no aspecto psicológico que inclui autopercepção da criança do seu próprio sorriso e o aspecto social que inclui a utilização do sorriso para demonstração de sentimentos. No caso relatado, o paciente embora jovem valorizava a questão estética e solicitou a reabilitação, fator importante a ser considerado, pois a autoimagem e a autoestima estão em formação e se não bem trabalhadas podem trazer consequências psicológicas para a vida adulta

Palavras chaves: Avulsão Dentária. Estética. Criança.

\section{Introdução}

Nos primeiros anos de vida, as crianças tornam-se mais vulneráveis a traumas dentários, pois estão desenvolvendo habilidades motoras e aprendendo a caminhar e socializar ${ }^{1}$. Em virtude disso, quedas na infância e choques inespecíficos se tornam comuns e em maior gravidade, podendo ocasionar injúrias na dentição decídua resultando na perda precoce de dentes decíduos ${ }^{2}$.

As injúrias traumáticas mais frequentes e citadas na literatura são a subluxação, a intrusão e a avulsão ${ }^{2,3}$ e suas consequências são relevantes e de extrema importância para cirurgiões-dentistas clínicos gerais e profissionais da área da saúde, uma vez que são estes os primeiros a se depararem com tal condição ${ }^{1,4,5}$. O traumatismo dentoalveolar continua sendo a razão mais frequente para consultas em dentistas ${ }^{2}$ e, em menor frequência, em unidades de pronto atendimento hospitalares ${ }^{4}$.

Os fatores que podem aumentar a frequência desses traumas são amplamente discutidos e cita$\operatorname{dos}^{3,4}$, e tem-se como principais causas a utilização da chupeta, um acentuado overjet e não selamento labial. Assim nestas situações os pais devem ser

Cirurgiã - Dentista, Aluna do Programa de Pós graduação - Área Odontopediatria, Departamento de Odontologia Social e Preventiva, da Universidade Federal de Pelotas, Pelotas, RS, Brasil.

Doutora em Odontopediatria pela UFPel (Pelotas, RS), Professora da disciplina de Odontopediatria na Faculdade Especializada na Área de Saúde do Rio Grande do Sul (FASURGS), Passo Fundo, RS.

*** Mestre em Odontopediatria pela SLMandic (Campinas, SP), Professora da disciplina de Odontopediatria na Faculdade Especializada na Área de Saúde do Rio Grande do Sul (FASURGS), Passo Fundo, RS, Brasil.

**** Doutora em Odontopedaitria pela SLMandic (Campinas, SP), Professora da disciplina de Odontopediatria na Faculdade Especializada na Área de Saúde do Rio Grande do Sul (FASURGS), Professora da disciplina de Odontopediatria na Universidade de Passo Fundo (UPF), Passo Fundo, RS, Brasil.

****** Mestre em Odontologia pela UPF (Passo Fundo, RS), Professora da disciplina de Ortodontia na Faculdade Especializada na Área de Saúde do Rio Grande do Sul (FASURGS), Passo Fundo, RS, Brasil. 
alertados e, dessa forma, a possibilidade de ocorrência de traumas pode ser minimizada ${ }^{4,5}$.

Existem situações em que condutas preventivas são irrelevantes, como no caso de uma queda da altura de uma laje de uma casa, situação esta que não pode ser evitada apesar da atenção dos familiares. As principais causas são que muitas crianças ainda estão aprendendo a andar, além do fato de gostarem de ver a vida com outra perspectiva, com olhos de um super-herói, sempre sobre árvores, móveis, muros e lajes ${ }^{6}$, e as crianças tem a probabilidade de queda aumentada porque as características do corpo favorecem esse tipo de acidente ${ }^{6,7}$, pois o tamanho e o peso da cabeça em relação ao corpo favorecem o desequilíbrio ${ }^{7}$.

Diante disso, setores de urgência hospitalar e pronto atendimento também devem estar aptos, e possuírem equipes preparadas para este tipo de atendimento ${ }^{8}$, pois as quedas representam a principal causa de atendimentos em serviços de emergência e de internações em crianças de zero a nove anos de idade. As consequências dependem da idade da criança, podendo ocasionar injúrias traumáticas, como fraturas faciais envolvendo avulsão dentária necessitando de atendimento cauteloso ${ }^{7,8}$.

Dessa forma devemos estar atentos ao modo como ocorrem os traumas na dentição decídua. Especial atenção deve ser dada às possíveis consequências para a dentição decídua e permanente e as condutas que devem ser tomadas para tranquilizar a criança, pais e cuidadores independente da situação do trauma, do local em que o trauma ocorreu e onde a criança foi atendida ${ }^{4,5}$. Nenhuma situação é tão traumática para o paciente e para seus familiares quanto a avulsão acidental de dentes anteriores, pois causa uma alteração estética muito alarmante, e causa impacto emocional e psicológico que envolve a criança e seus acompanhantes ${ }^{8-10}$.

O presente artigo objetiva discutir um caso clínico de avulsão precoce de dentes decíduos em criança com 2 anos e 5 meses com fratura mandibular unilateral ocorrida após uma queda de 3 metros de altura, situação extremamente delicada para realização de atendimento de trauma dentário infantil, exigindo habilidades para adoção dos primeiros socorros e tranquilizar os familiares. Como tratamento adotado utilizou-se um mantedor de espaço estético-funcional após a redução da fratura mandibular, já que a criança queria muito os dentes. Dessa forma, serão discutidos os aspectos inerentes e necessários ao sucesso do tratamento estabelecido.

\section{Relato de caso}

Paciente do gênero masculino, leucoderma, 2 anos e 5 meses de idade, apresentou-se na emergência do Hospital São Vicente de Paulo na cidade de Passo Fundo - RS com história de queda de 3 metros de altura da laje da casa que estava em reforma.
Ao exame físico intra-oral, observou-se ausência dos elementos dentários 51 e 61 devido a queda, e foi observada equimose em região mandibular unilateral, assimetria facial, má oclusão e mobilidade óssea características compatíveis com fratura mandibular. Não havia edema ou supuração no local. Clinicamente, o paciente apresentava a dentição decídua incompleta com ausência de lesões de cárie. $\mathrm{Na}$ anamnese, a responsável relatou que o paciente não apresentava alterações sistêmicas, dificuldade de alimentação, bem como ausência de vômitos e perda da consciência.

Após análise da tomografia computadorizada e exames radiográficos confirmou-se diagnóstico de fratura deslocada de corpo mandibular esquerdo. O plano de tratamento foi explicado aos pais que consentiram com o tratamento e assinaram o prontuário de atendimento, o qual continha também a autorização para a publicação do caso. O tratamento foi realizado em caráter de urgência, com acesso intrabucal às fraturas sob anestesia geral. As fraturas foram reduzidas e estabilizadas por meio de odontossíntese com fio de aço. A oclusão foi estabilizada manualmente e, em seguida, foram instaladas miniplacas de titânio na borda inferior da mandíbula seguida de sutura com fios reabsorvíveis.

Após receber alta hospitalar, o paciente permaneceu sob acompanhamento ambulatorial mantendo boa cicatrização. Foi mantida orientação de dieta pastosa e repouso físico por um período de 30 dias. As radiografias pós-operatórias evidenciaram fixação satisfatória e após 1 ano as placas foram removidas por meio de acesso intrabucal.

Após a redução da fratura da mandíbula, optou-se pela reabilitação protética com mantedor de espaço estético-funcional. Após um mês da alta dada pela equipe cirúrgica do hospital, foi realizada radiografia panorâmica e modelos de estudo para planejamento e confecção do mantedor de espaço. Assim que realizado o plano de tratamento, partiu-se para o tratamento propriamente dito. Na primeira fase clínica, foi realizada a escolha das bandas e moldagem de transferência. Para a fase laboratorial, o modelo com as bandas foi encaminhado ao laboratório para a confecção do mantedor fixo do tipo botão de Nance (aparelho ortodôntico com ancoragem dento-muco suportada, utilizado como mantenedor de espaço, indicado para perdas múltiplas), com um parafuso expansor do tipo Hyrax na linha média para garantir a manutenção do espaço e permitir o crescimento ósseo maxilar, e dentes de acrílico presos ao fio ortodôntico soldado às bandas (Figuras 1, 2 e 3). Todo o processo de confecção do aparelho foi realizado em laboratório especializado após a discussão entre o dentista e o protético. $\mathrm{Na}$ segunda fase clínica o mantenedor de espaço foi instalado. Usou-se cimento de ionômero de vidro para cimentação para cimentar as bandas ortodônticas nos molares decíduos (Figura 4). 


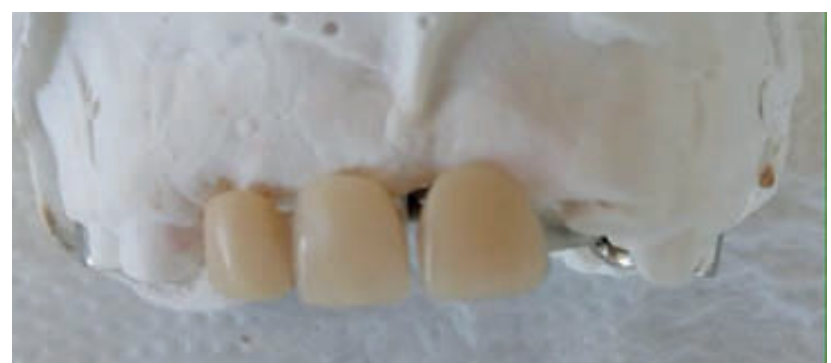

Figura 1 - Vista frontal do aparelho mantenedor de espaço estético-funcional

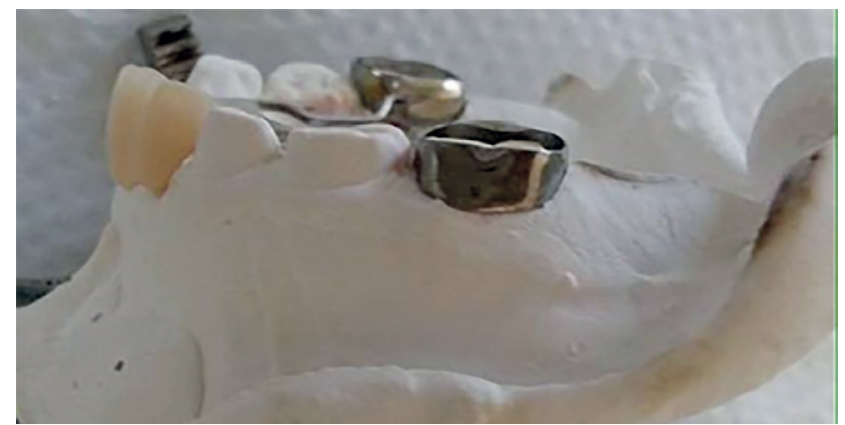

Figura 2 - Vista lateral do aparelho mantenedor de espaço estético-funcional

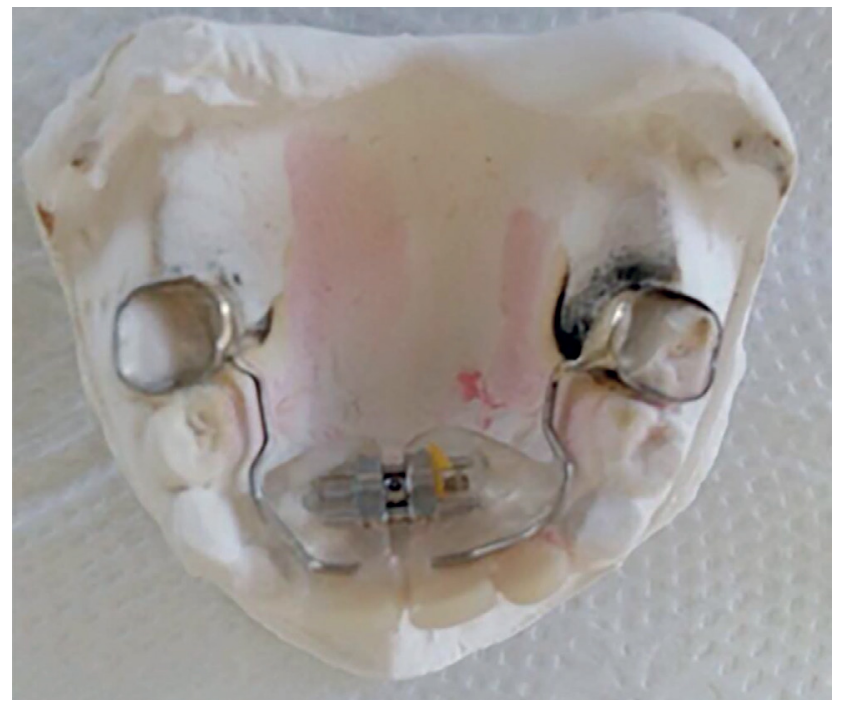

Figura 3 - Vista oclusal do aparelho mantenedor de espaço estético-funcional

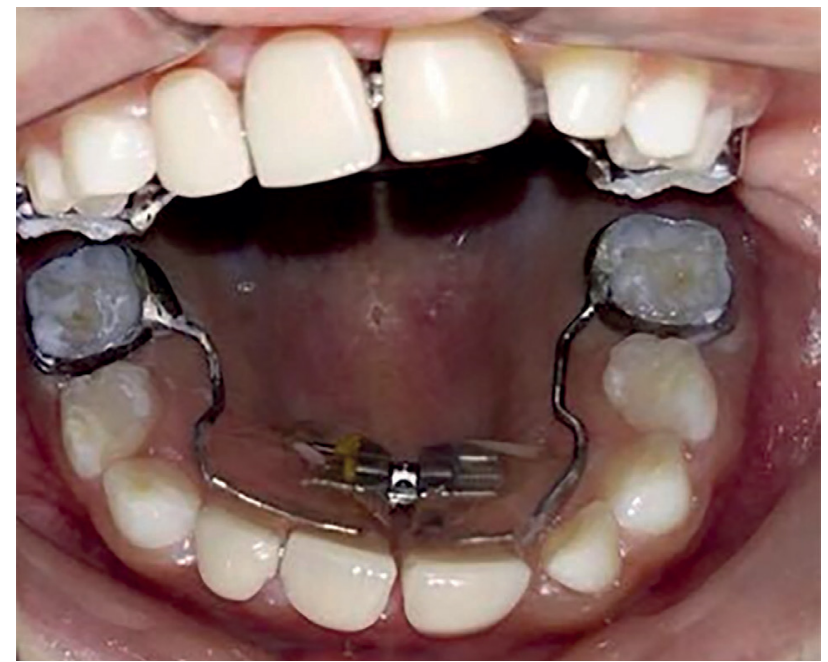

Figura 4-Aparelho mantenedor de espaço estético-funcional cimentado
Nas consultas de acompanhamento, percebeu-se a desadaptação do acrílico e a mobilidade excessiva do elemento 52, que foi extraído (Figura 5) e então realizou-se nova moldagem e encaminhou-se o aparelho novamente ao laboratório para a inclusão de mais um elemento no novo mantedor confeccionado (Figura 6). A ativação do parafuso era feita pelo cirurgião-dentista nas consultas de acompanhamento. Ativava-se $1 / 4$ de volta a cada 6 meses para acompanhar o crescimento ósseo. A remoção definitiva do mantedor deu-se quando os primeiros dentes decíduos começaram a esfoliar.

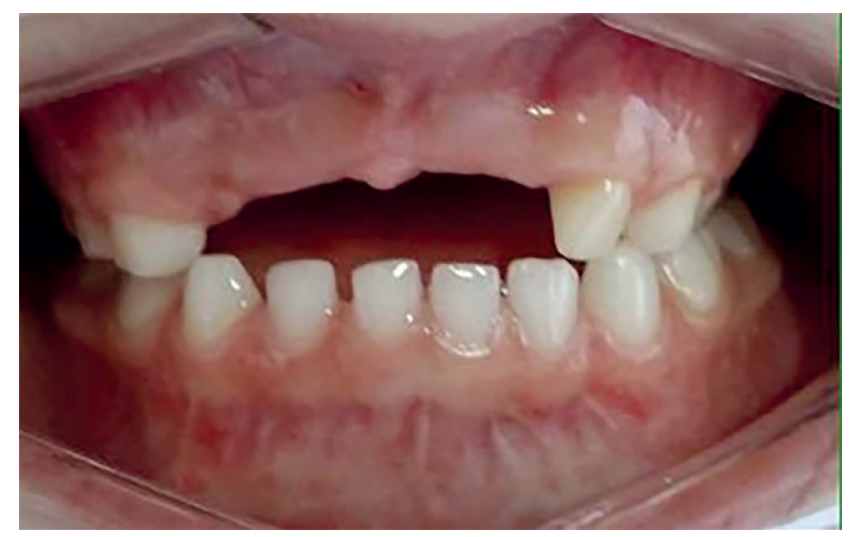

Figura 5 - Dente 52 extraído.

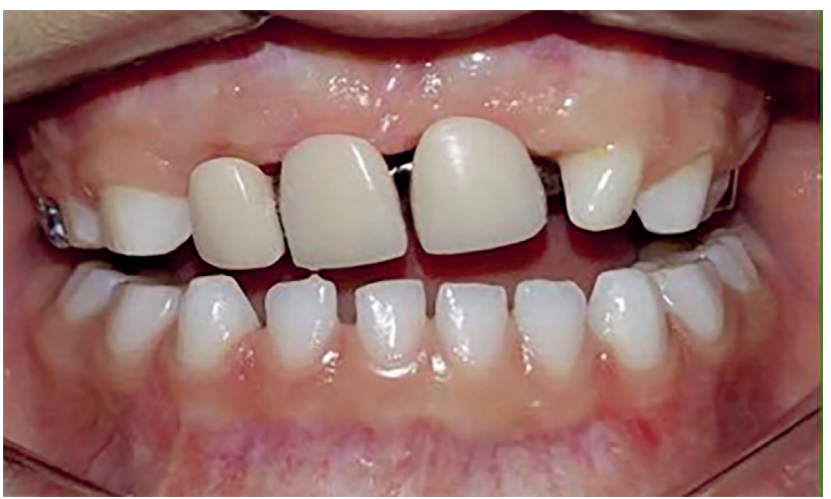

Figura 6 - Resultado final do mantenedor de espaço

\section{Discussão}

Traumatismo dentário é um problema frequente durante os primeiros anos de vida das crianças ${ }^{1,3,4}$ e a perda precoce dos dentes decíduos anteriores é relativamente comum. O traumatismo dentoalveolar é a segunda razão mais frequente de visitas aos dentistas $^{3,4}$, e o trauma facial tornou-se um assunto inevitável para os médicos devido a sua frequência ser cada vez maior nas quatro ultimas décadas ${ }^{7}$, auxiliando no primeiro atendimento ao paciente, na conduta correta frente ao trauma e na agilidade para encaminhar o caso ao especialista, situações importantes para o prognóstico ${ }^{1}$.

De acordo com estudos, a prevalência dos traumas dentários varia entre $4 \%$ e $32 \%$, e esta dife- 
rença é atribuída a diferenças nos fatores avaliados como o tipo de lesão, tipo de estudo e população analizada $^{11,12}$. Diferentes autores identificaram que a idade de 1 a 4 anos é mais vulnerável a este tipo de trauma ${ }^{12}$, sendo o dente incisivo central superior o mais envolvido, seguido do incisivo lateral superior e caninos ${ }^{3}$.

Nos acidentes de queda de altura que envolvem crianças, os traumas mais frequentes são fraturas mandibulares ${ }^{7}$ e os incisivos centrais superiores estão entre os dentes mais acometidos, pois estes elementos se localizam na posição anterior da arcada, podem se encontrar em protrusão e muitas vezes com cobertura inadequada dos lábios superiores tornando-os mais favoráveis ao traumatismo ${ }^{2,8}$.

Dessa forma, as reabilitações tornam-se importantes, pois a perda dentária causa impacto no estado da saúde bucal do paciente e na sua qualidade de vida, incluindo bem-estar social e bem-estar emocional ${ }^{13,14}$. Diante disso, substituir os dentes decíduos perdidos precocemente por aparelhos protéticos é de grande importância, pois atenuam os resultados de um trauma e recuperam a autoestima do paciente e familiares ${ }^{5}$.

Estudos demostram que o trauma alveolodentar tem um impacto negativo e influenciam diretamente na qualidade de vida das crianças e familiares $^{8,12,13}$. Kramer et al. ${ }^{12}$ (2013) encontraram em seu estudo que o trauma é a segunda causa, após cárie dentaria, que causa impacto negativo na qualidade de vida das crianças, e que este impacto chega a ser $70 \%$ maior quando comparado com as que não sofreram trauma. Diante disso percebemos a importância de estabelecer prioridades nas reabilitações, utilizando-as como uma estratégia para diminuir o impacto negativo que a injuria causa na qualidade de vida ${ }^{15}$. Por tal motivo, optou-se pela reabilitação precoce através de um mantenedor de espaço estético-funcional que permite o crescimento facial e devolve o anteparo lingual que os dentes anteriores proporcionam, além de restabelecer a estética desejada pelo paciente e pela família.

Outro motivo para realização do tratamento após perda dental precoce é que ele também inclui o aspecto funcional dos dentes e da cavidade oral, como a mastigação ${ }^{14}$ e a habilidade correta de emitir os sons, pois o desenvolvimento da fala depende de muitos fatores, entre os quais, a presença de dentes antero-superiores ${ }^{13-15}$. Tendo-se em vista a idade da criança torna-se relevante a reabilitação, pois a fala ainda está se desenvolvendo e a falta dos dentes anteriores pode acarretar em um pronúncia alterada de alguns fonemas ${ }^{12}$.

A utilização de mantedor de espaço estético-funcional para substituir incisivos perdidos precocemente é uma alternativa condizente e de extrema importância para o desenvolvimento do equilíbrio oral da criança, para manter o equilíbrio neuromuscular, a estética e as funções de fonação, mastigação, deglutição. Toda vez que houver perda de dentes anteriores e o paciente apresenta-se apto, indica-se a instalação de um aparelho mantenedor de espaço funcional ${ }^{16}$. Já que estudos contraindicam o reimplante como tratamento para dentes decíduos avulsionados.

A reabilitação é importante, pois o sorriso é uma das formas mais eficazes para que as pessoas transmitam suas emoções e fundamental para o convívio social ${ }^{20}$. As crianças se comunicam com ou sem ele, quando estão felizes ou tristes, confiantes ou incertos ${ }^{5}$. A ausência dos padrões de sorriso das crianças influenciam a saúde e qualidade oral de vida de duas maneiras, no aspecto psicológico que inclui auto-percepção da criança do seu próprio sorriso e no aspecto social, que inclui o a utilização do sorriso para demonstração de sentimentos ${ }^{11,12}$. No caso relatado, o paciente embora jovem valorizava a questão estética e solicitou a reabilitação, fator importante a ser considerado, pois a autoimagem e a autoestima estão em formação e se não bem trabalhadas podem trazer consequências psicológicas para a vida adulta

Devido ao desejo de reabilitação, tanto por parte dos pais quando da criança, optou-se pela confecção de uma aparelho mantedor de espaço fixo, tipo barra palatina com parafuso expansor. Estes são indicados para pacientes com perdas de um ou mais dentes e estão entre os aparelhos mais utilizados devido a sua fácil confecção e manejo, além de restaurar a oclusão funcional, a estética, permitem uma fácil higienização ${ }^{20,21}$.

Os pais e a criança foram informados sobre o funcionamento do aparelho e sua finalidade, sobre os cuidados de higienização e com a alimentação, sobre o aumento do risco de cárie e do alojamento de restos de alimentos entre os dentes. Também foram informados da necessidade de acompanhamento clínico e radiográfico.

\section{Considerações Finais}

A perda precoce de dentes decíduos e suas consequências são um tema relevante, influenciam na qualidade de vida das crianças e a reabilitação se faz necessária para evitar problemas como fonação, deglutição.

O sorriso é uma das manifestações mais eficazes para a transmissão de emoções e para a comunicação social. A ausência dos padrões de sorriso das crianças influenciam a saúde e qualidade oral de vida no aspecto psicológico e no aspecto social. No caso relatado, o paciente valorizava a própria aparência e solicitou a reabilitação, fator importante a ser considerado, pois a autoimagem e a autoestima estão em formação. Essas questões, se não bem trabalhadas, podem trazer consequências psicológicas para a vida adulta. 


\section{Abstract}

Objective: to discuss the rehabilitation of a clinical case of early avulsion of deciduous teeth and unilateral mandibular fracture occurred after a three-meter fall. Case report: male patient, leucoderma, 2 years and 5 months old, presented at the Emergency Room of Hospital São Vicente de Paulo in the city of Passo Fundo, RS, Brazil, with history of falling from the slab of his house that was under renovation. The intraoral physical examination showed the absence of teeth 51 and 61, bruise on the unilateral mandibular region, and mandibular fracture. A maintainer of aesthetic and functional space was adopted as treatment after the reduction of mandibular fracture. Final considerations: the absence of smile patterns for children influences the health and oral quality of life in two ways: the psychological aspect, which includes self-perception of children on their own smile; and the social aspect, which includes using the smile to show feelings. In the case reported, although the patient was young, he valued aesthetics and requested rehabilitation. This is a major factor for consideration, because self-image and self-esteem are under development, and if not well managed, they may have psychological consequences in adult life.

Keywords: Tooth Avulsion. Aesthetics. Child.

\section{Referências}

1. Cunha RF, Pugliesi DMC, Vieira AEM. Oral trauma in Brazilian patients aged 0-3 years. Dent Traumatol 2001;17(5):210-2.

2. Jihan Turkistani1, Azzah Hanno. Recent trends in the management of dentoalveolar traumatic injuries to primary and young permanent teeth. Dent Traumatol 2011; 27(1): 46-54.

3. Mendoza-Mendoza A, Iglesias-Linares A, Yanez-Vico RM, Abalos-Labruzzi C. Prevalence and complications of trauma to the primary dentition in a subpopulation of Spanish children in southern Europe Asuncion. Dent Traumatol 2015; 31(2): 144-149.

4. Mahmoodi B, Rahimi-Nedjat R, Weusmann J, Azaripour A, Walter C, Willershausen B. Traumatic dental injuries in a university hospital: a four-year retrospective study. BMC Oral Health 2015; 139(15):1-7.

5. Iso-Kungas P, Suominen AL, Lindqvist C, Thorén H. Dental Injuries in Pediatric Patients With Facial Fractures Are Frequent and Severe. J Oral Maxillofac Surg 2012; 70(2): 396-400.

4. Kramer PF, Feldens EG, Bruch CM, Ferreira SH, Feldens CA. Clarifying the effect of behavioral and clinical factors on traumatic dental injuries in childhood: a hierarchical approach. Dent Traumatol 2015; 31(3): 177-183.

5. Patel RR, Tootla R, Marita R, Inglehart M. Does oral health affect self perceptions, parental ratings and video- based assessments of children's smiles? Community Dent Oral Epidemiol. 2007;35(1):44-52.

6. Lucht UA. A prospective study of accidental falls and resulting injuries in the home among elderly people. Acta Soc Med Scand 1971;2:105-9.

7. Montovani JC, Campos LMP, Gomes MA, Moraes VRS, Ferreira FD, Nogueira EA. Etiologia e incidência das fraturas faciais em adultos e crianças: experiência em 513 casos. Rev Bras Otorrinolaringol 2006;72(2):235-41.
8. Dang KM, Day PF, Calache H, Tham R, Parashos P. Reporting dental trauma and its inclusion in an injury surveillance system in Victoria, Australia. Australian Dent Journ 2015; 60(1): 88-95.

9. Wilson CFG. Manangement of trauma to primary and developing teeth. Dent Clin North Am 1995; 39(1):133-167.

10. Rulli MA. Aspectos biológicos dos reiroplantes dentários. Uma síntese. Rev Assoe Paul Cir Dent 1979; 33(6):482-487.

11. Bhardwaj VK, et al. Prevalence of anterior teeth fracture among institutionalized visually impaired individuals in Himachal Pradesh, India - A cross-sectional study. Journ of Cranio-Maxillary Diseases 2015; 4(2): 117-122.

12. Kramer PF, Feldens CA, Ferreira SH, Bervian J, Rodrigues $\mathrm{PH}$, Peres MA Exploring the impact of oral diseases and disorders on quality of life of preschool children. Community Dent Oral Epidemiol 2013; 41(4): 327-335.

13. Firmino RT, Gomes MC, Clementino MA, Martins CC, Painva SM, Granville-Garcia AF. Impact of oral health problems on the quality of life of preschool children: a case-control study. Intern Journ of Paediat Dent 2016; 26(4):242-249.

14. Golai S, Nimbeni B, Devipatil S, Baali P, Kumar H. Impact of Untreated Traumatic Injuries to Anterior Teeth on the Oral Health Related Quality of Life As Assessed By Video Based Smiling Patterns in Children. J of Clin and Diagnost Resear 2015; 9(6): ZC16-ZC19.

15. Aldrigui JM, Abanto J, Carvalho TS, Mendes FM, Wanderley MT, Bonecker M et al. Impact of traumatic dental injuries and malocclusions on quality of life of young children. Health Qual Life Outcomes 2011; 78(9): 1-44.

16. Camargo FG, et al. Traumatismo em crianças na fase da dentição decídua. Odonto/ USF 1996; 14 (1):113-128.

17. Lam R. Epidemiology and outcomes of traumatic dental injuries: a review of the literature. Australian Dental Journal 2016; 61(1): 4-20.

18. Leao A, Sheiham A. Relation between clinical dental status and subjective impacts on Daily Living. J Dent Res. 1995;74(7):1408-13.

19. Jokovic A, Locker D, Stephens M, Kenny D, Tompson B, Guyatt G. Validity and eliability of a questionnaire for measuring child oral-health-related quality of life. J Dent Res 2002; 81(8):459-63.

20. Pereira L, Miasato JM. Mantenedor de Espaço Estético-funcional em Odontopediatria. Rev de Odontol da Univ Cidade de São Paulo 2010; 22(2): 154-62.

21. Diniz MB, Silva RC, Zuanon ACC. Perda dental precoce e manutenção de espaço na dentadura decídua: relato de um caso clínico. Rev Ibero-am Odontopediatr Odontol Bebê 2005; 8(44):376-81.

Endereço para correspondência:

Gabriela dos Santos Pinto

R. Angélica Oto, 160 - Boqueirão

99025-270 Passo Fundo - RS

Fone: (54) 3335-8900

E-mail: gabipinto@hotmail.com

Recebido: 07/10/2016. Aceito: 02/02/2017. 